\title{
Growth and Surface Reconstructions of AIN(0001) Films
}

C. D. Lee ${ }^{1 *}$, Y. Dong ${ }^{1}$, R. M. Feenstra ${ }^{1}$, J. E. Northrup ${ }^{2}$, J. Neugebauer ${ }^{3}$

${ }^{1}$ Dept. Physics, Carnegie Mellon University, Pittsburgh, PA 15213

${ }^{2}$ Palo Alto Research Center, 3333 Coyote Hill Road, Palo Alto, California 94304

${ }^{3}$ University of Paderborn, Warburger Str. 100, 33098 Paderborn, Germany

\begin{abstract}
The growth and surface reconstructions of AlN(0001) films were studied. For moderately Alrich surfaces, the $2 \times 6$ structure is commonly observed in reflection high energy electron diffraction. It is found that this pattern consists of $2 \sqrt{ } 3 \times 2 \sqrt{ } 3-\mathrm{R} 30^{\circ}$ and $5 \sqrt{ } 3 \times 5 \sqrt{ } 3-\mathrm{R} 30^{\circ}$ reconstructions according to scanning tunneling microscopy. Similar to the Ga-rich $\mathrm{GaN}(0001)$ surface, these structures are determined to contain 2-3 monolayers of excess Al terminating the surface. Based on first-principles theory the structures are believed to contain a laterally contracted Al layer. At higher Al coverage a thick, flat $\mathrm{Al}$ film is found to form on the surface. A high density of growth spirals (associated with threading dislocations having full or partial screw character) is found to be present on the surface, although this density decreases with increasing growth temperature.
\end{abstract}

\section{INTRODUCTION}

AlN has been considered as a useful material for high temperature and high power electronic devices and also optoelectronic device applications. Since the lattice constant of AIN is relatively closely matched to that of $\mathrm{SiC}(+0.9 \%)$, it has been used as a buffer layer for high power electronic devices. In contrast to recent progress in identifying surface structural arrangements on GaN [1], there is only limited knowledge currently on the surfaces structures of AlN. Several studies have previously reported the symmetry of AlN surface reconstructions as seen by reflection high energy electron diffraction (RHEED) [2-9]. RHEED patterns with symmetries of $1 \times 1$ and $2 \times 2$ are commonly reported, a $2 \times 6$ pattern is also commonly seen, and a sequence consisting of $1 \times 3,3 \times 3$, and $6 \times 6$ is reported by several groups. (For RHEED on a surface with hexagonal symmetry, a $1 \times 3$ pattern corresponds to a $\sqrt{3} \times \sqrt{3}-\mathrm{R} 30^{\circ}$ symmetry.) Structural arrangements associated with these reconstructions have not been determined. In this work we report on scanning tunneling microscopy (STM), reflection high energy electron diffraction (RHEED) and low energy electron diffraction (LEED) measurements of reconstructions of the AlN(0001) surface.

\section{EXPERIMENTAL}

AlN films were grown by plasma-assisted molecular beam epitaxy (PAMBE) as previously described [10]. In order to minimize surface roughening due to the $-2.5 \%$ lattice mismatch of AlN relative to $\mathrm{GaN}$, a sequence containing $\approx 1 \mathrm{~nm}$-thick AlN layers followed by $\approx 2 \mathrm{~nm}$-thick Sidoped GaN layers was deposited. An undoped AlN layer with thickness of about $10 \mathrm{~nm}$ was then deposited on top. Somewhat flatter morphology was obtained in this case compared to direct deposition of AlN on GaN. A growth temperature of $750-800^{\circ} \mathrm{C}$ was generally used although on a few occasions we have occasionally used higher growth temperatures. In a few instances AlN 
was directly deposited on $6 \mathrm{H}-\mathrm{SiC}$; a flat morphology was also achieved in that case, and the surface reconstructions as observed by RHEED were the same as for the AIN deposition on GaN. After the growth the samples were transferred under ultra high vacuum to an adjoining analysis chamber for study by STM and LEED.

\section{RESULTS \& DISCUSSION}

During MBE growth under Al-rich conditions the AlN surfaces display a $1 \times 1$ RHEED pattern. After the growth, the $1 \times 1$ (Al-rich) RHEED pattern generally persists during the cool down. Occasionally a $2 \times 6$ RHEED pattern can result directly from this type of growth termination, and if not, it can be obtained by performing a post-growth anneal at $800^{\circ} \mathrm{C}$ for about $10 \mathrm{~min}$. The reconstruction pattern appears when the substrate temperature is at $400-500^{\circ} \mathrm{C}$ which is below the melting point of Al. Somewhat longer annealing times produce a surface with mixed $2 \times 6$ and $1 \times 3$ character, and still longer annealing results in a $1 \times 1$ pattern.

Figure 1(a) shows a STM image for a surface with a $2 \times 6$ RHEED pattern. The terraced surface consists of the long-period and short-period (lower left-hand corner) structures. A hexagonal corrugation appears on the surface with measured separation between corrugation maxima of $25 \pm 3 \AA$ for long-period and $10 \pm 1 \AA$ for short-period structures, respectively. We note that the orientations of the short-period and long-period corrugation patterns are identical. In LEED as shown in Fig. 1(b), the six first-order spots are apparent, with spacing relative to the
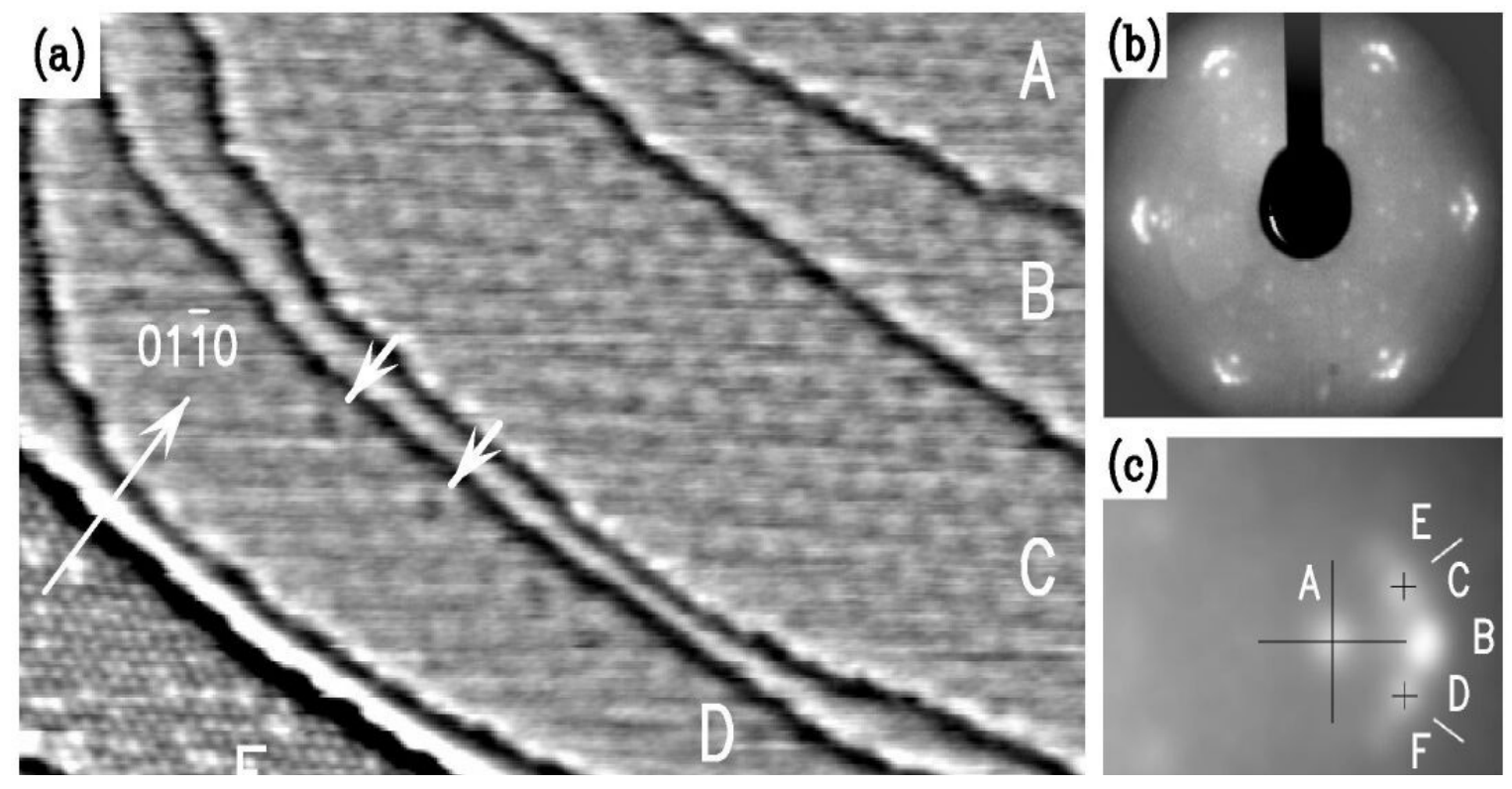

Fig. 1. (a) STM image of a surface with multiple reconstructions, acquired with sample voltage of $+3 \mathrm{~V}$. The image is displayed using a background subtraction in which the background is formed by averaging the image over a window of $3 \times 3 \mathrm{~nm}^{2}$. A region of $2 \sqrt{ } 3 \times 2 \sqrt{ } 3-\mathrm{R} 30^{\circ}$ structure is seen in bottom left-hand corner, and remainder of the terraces consists of $5 \sqrt{ } 3 \times 5 \sqrt{ } 3$ $\mathrm{R} 30^{\circ}$ structure. (b) LEED pattern, acquired at $80 \mathrm{eV}$ electron energy. The left-most first-order peak is slightly distorted due to a stray light reflection in the optical system used to acquire the image. (c) Expanded view of first-order reflection and satellite peaks. 
origin of $b=2 /(\sqrt{3} a)$ and located along $\langle 1 \overline{1} 00\rangle$ directions. At smaller wavevectors, additional spots with $b /(2 \sqrt{3})$ spacing and located along both $\langle 11 \overline{2} 0\rangle$ and $\langle 1 \overline{1} 00\rangle$ directions are seen. From this arrangement it is clear that an underlying $2 \sqrt{ } 3 \times 2 \sqrt{3}-\mathrm{R} 30^{\circ}$ symmetry is present in the pattern. We conclude that the basic symmetry of the surface reconstructions is rotated by $30^{\circ}$ relative to the underlying $1 \times 1$ lattice. The observed corrugation with $10 \pm 1 \AA$ spacing would thus correspond to a $2 \sqrt{3} \times 2 \sqrt{3}-\mathrm{R} 30^{\circ}$ symmetry (this surface periodicity will produce a $2 \times 6$ pattern in RHEED). For the long-period structure, with period 2.5 \pm 0.04 times larger than the short-period one and the same orientation, we identify it as $5 \sqrt{3} \times 5 \sqrt{ } 3-\mathrm{R} 30^{\circ}$. Furthermore, based on Auger electron spectroscopy, we conclude that this surface has an Al coverage between 2 and 3 monolayers (ML). Detailed analysis of the LEED patterns have been discussed elsewhere [11]. The surface structure appears to contain a laterally contracted metallic overlayer of $\mathrm{Al}$, similar to that of the pseudo- $1 \times 1$ structure of $\mathrm{GaN}[1]$.

Figure 2 shows large-scale STM images, illustrating the general morphology of the AlN surfaces prepared under moderate Al-rich conditions. For growth temperatures of $780^{\circ} \mathrm{C}$ a large number of growth spirals are present on the surface as shown in Fig. 2(a). These growth spirals form around threading dislocations with full or partial screw character, thus revealing a density of these defects of approximately $3 \times 10^{9} \mathrm{~cm}^{-2}$ for these films. This value is much greater than the threading screw dislocation density of $\approx 1 \times 10^{8} \mathrm{~cm}^{-2}$ found typically on the MOCVD-grown GaN films used as our substrates. For the film grown at $860^{\circ} \mathrm{C}$, we found that the number of screw dislocations in the film is significantly reduced, to about $3 \times 10^{8} \mathrm{~cm}^{-2}$ as shown in Fig. 2(b). This result indicates that in order to minimize the screw type dislocation density the high temperature growth for the AlN is desirable. Samples grown at even higher temperatures, near $900^{\circ} \mathrm{C}$, display rough surfaces.
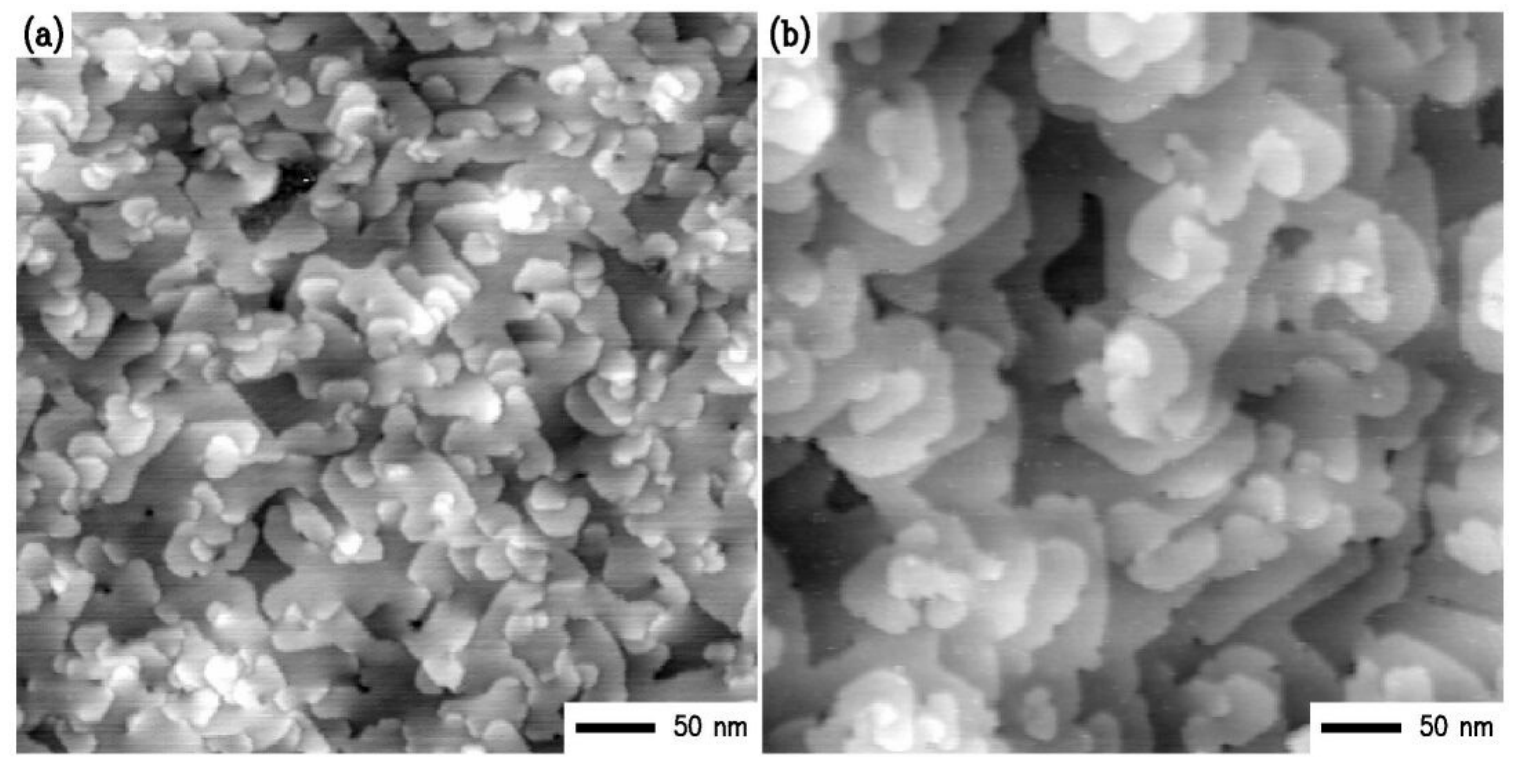

Fig. 2. Large-scale STM images of Al-rich $A \mathrm{AN}(0001)$ surface with different substrate temperature. (a) $\mathrm{T}_{\text {sub }}=780{ }^{\circ} \mathrm{C}$, sample voltage $+1.5 \mathrm{~V}$ and displayed with gray scale range of $10 \AA$. (b) $\mathrm{T}_{\text {sub }}=860^{\circ} \mathrm{C}$, sample voltage is $+1.0 \mathrm{~V}$ and gray scale range is $12 \AA$. 
Turning to results for higher Al coverage, Fig. 3(a) shows a RHEED pattern of the surface covered by a thick $(\approx 5 \mathrm{ML}) \mathrm{Al}$ layer. These films are formed by deposition of $\mathrm{Al}$ on the asgrown AIN surface, at a temperature of about $150^{\circ} \mathrm{C}$. The resulting surface morphology is flat, with no Al droplets [11]. This result is in contrast to the situation for Ga on $\mathrm{GaN}(0001)$, in which deposition of $\mathrm{Ga}$ at temperature of $20^{\circ} \mathrm{C}$ or above does result in the formation of Ga droplets. For our AIN(0001) surfaces we find that the excess Al forms droplets for deposition (or annealing) at a temperature of $750^{\circ} \mathrm{C}$, in agreement with prior reports $[12,13]$. We have not studied in detail the temperature dependence of the droplet formation (i.e. at what temperature between 150 and $750^{\circ} \mathrm{C}$ they first form), but in any case it appears that their formation is kinetically limited at the low temperatures.

The RHEED pattern of Fig. 3(a) displays narrow first-order streaks, but at wavevectors distinctly higher than for the AIN $1 \times$ streaks (the former are indicated by dashed lines in the RHEED patterns of Fig. 3 and the latter by solid lines). We refer to this type of pattern obtained from thick $\mathrm{Al}$ films as $1 \times 1-\mathrm{Al}$. Thermal annealing at temperatures above about $750^{\circ} \mathrm{C}$ for $5-10$ minutes is found to effectively remove this thick $\mathrm{Al}$ film from the surface; the RHEED pattern of Fig. 3(b) was acquired from such an annealed surface, maintained at the elevated temperature. With sufficient annealing time the A1/N Auger ratio returns to a value close to that of the $2 \times 6$ structure (Fig 3(b)), and the RHEED pattern displays the $2 \times 6$ symmetry. In contrast, Fig. 3(d) shows the pattern obtained from a surface which started with the thick Al film and was then
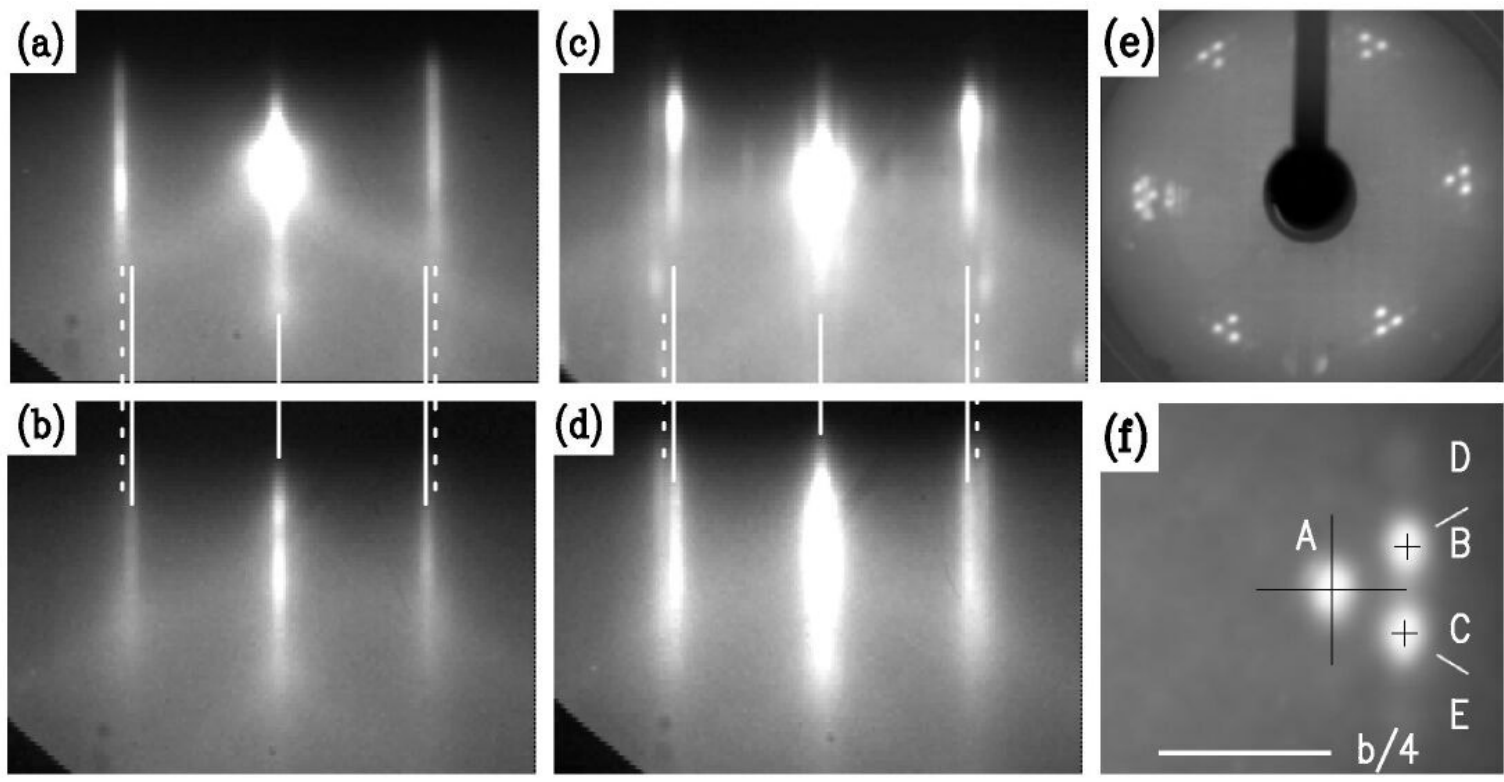

Fig. 3. (a)-(d) RHEED images with electron beam along (11 $\overline{2} 0$ ): (a) $20^{\circ} \mathrm{C}$, surface covered by $\approx 5 \mathrm{ML}$ thick $\mathrm{Al}$ film; (b) $750^{\circ} \mathrm{C}, 15 \mathrm{~min}$ anneal; (c) $20^{\circ} \mathrm{C}$, following cool down as same surface as in (a); (d) $20^{\circ} \mathrm{C}$, following cool down of a similar surface as in (b) but that was annealed for only 10 minutes. White solid lines indicate the location of 1 st order AIN streaks, and dashed lines indicate 1st order streaks associated with the thick Al overlayer. (e)-(f) LEED patterns, acquired at $80 \mathrm{eV}$ electron energy, of $\mathrm{Al}$ films with thickness of a few ML deposited on AIN(0001). Panel (f) shows expanded views of the first-order reflections and satellite peaks from (e). 
annealed for a time insufficient to completely remove the excess Al. The result is something which is clearly not $2 \times 6$, but rather, displays streaks at the $1 \times$ locations of AlN and neighboring streaks at the locations corresponding to the thick Al film. The former streaks are marked by the solid white lines in Fig. 3, and the latter by the dashed white lines.

LEED patterns obtained from a surface displaying the $1 \times 1-A 1$ RHEED pattern are shown in Fig. 3(e) and (f). We observe a three-fold splitting of the first-order LEED spots, with satellite features $\mathrm{B}$ and $\mathrm{C}$ having essentially equal intensity to the primary A spot. Careful measurement of the spot positions indicated that the A spot is at a wavevector corresponding to the $1 \times 1 \mathrm{AlN}$ lattice (i.e. the same position as the first-order spots in Figs. 1(b) and (c)) [11]. Regarding the origin of the threefold splitting seen in the LEED, we believe that it indicates a distortion of the overlying Al layers such that the average Al-Al spacing is close to that of a close-packed $\mathrm{Al}(111)$ plane (spacing of $2.86 \AA$ compared with $3.11 \AA$ for AlN) [11]. In RHEED this detailed distortion is not apparent and we see only the average spacing of the Al atoms in the overlayer because the coherence length in RHEED is much greater than that of LEED [11].

First-principles total energy calculations have been performed for a large number of possible AIN reconstructions. The relative energies of the structures are determined as a function of the Al chemical potential, as discussed elsewhere $[11,14]$. For Al-rich conditions it is found that energetically favorable structures can be formed in which the top Al layer is laterally contracted by a factor of $\sqrt{ } 3 / 2$ and rotated by $30^{\circ}$ relative to the underlying layer [11]. These structures, which can form with either $4 / 3$ or $7 / 3$ ML of excess A1, have a natural symmetry of $\sqrt{ } 3 \times \sqrt{ } 3-\mathrm{R} 30^{\circ}$. The latter $7 / 3 \mathrm{ML}$ structure has an Al coverage close to that of the experimentally observed $2 \sqrt{ } 3 \times 2 \sqrt{ } 3-\mathrm{R} 30^{\circ}$ structure. Perhaps some type of symmetry lowering distortion of the model, such as a buckling of the Al surface atoms that relieves strain, may produce an arrangement with the required $2 \sqrt{ } 3$ symmetry. For the $5 \sqrt{ } 3 \times 5 \sqrt{ } 3$-R $30^{\circ}$ structure the size of the unit cell is much too large for the first-principles theory to handle, and future work with approximate potentials is needed to further study its structure.

Finally, for Al-poor conditions, reconstructions with symmetry of $2 \times 2$ and $1 \times 3$ have been observed in RHEED. As discussed earlier the $1 \times 3$ RHEED pattern is indicative of a $\sqrt{3} \times \sqrt{3}-\mathrm{R} 30^{\circ}$ reconstruction. The best candidate for this reconstruction that has emerged so far from the theoretical work is the 4/3 ML laterally contracted structure. For the $2 \times 2$ structure an energetically favorable model is an arrangement of $\mathrm{N}$ adatoms in $\mathrm{H}_{3}$ sites [11,14].

\section{CONCLUSION}

We have investigated the growth and surface reconstruction of $\operatorname{AlN}(0001)$. A characteristic $2 \times 6$ RHEED pattern was observed for Al coverage of 2-3 monolayers, and from LEED and STM it is found that this pattern originates from $2 \sqrt{3} \times 2 \sqrt{3}-\mathrm{R} 30^{\circ}$ and $5 \sqrt{3} \times 5 \sqrt{3}-\mathrm{R} 30^{\circ}$ surface structures. Based on first-principles theory it is argued that such structures likely incorporate a laterally contracted monolayer or bilayer structure containing a 4/3 ML Al layer contracted and rotated by $30^{\circ}$ such that it fits on the underlying $1 \times 1$ lattice. For Al films with thickness greater than a few ML we observe a characteristic $1 \times 1$ RHEED pattern but in which the diffraction streak spacing is about $5 \%$ greater than $1 \times 1$ AlN spacing, indicating a contraction of the lattice. 
We deduce that $\mathrm{Al}$ films exist on the surface with an atom density close to that of bulk Al. For lower coverage of $\mathrm{Al}$ we observe additional reconstructions with symmetry $2 \times 2$ and $1 \times 3$.

\section{ACKNOWLEDGEMENTS}

This work has been supported in part by the Office of Naval Research under grants N0001402-1-0933 (C. Wood) and N00014-02-0433 (L. Cooper). J.N. thanks the Deutsche

Forschungsgemeinschaft for financial support.

\section{REFERENCES}

* Present address: Raytheon RF Components, Andover, MA

[1] R. M. Feenstra, J. E. Northrup, and J. Neugebauer, MRS Internet J. Nitride Semicond. Res. 7, 3 (2002).

[2] M. A. L. Johnson, S. Fujita, W. H. Rowland, K. A. Bowers, W. C. Hughes, Y. W. He, N. A. El-Masry, J. W. Cook, J. F. Schetzina, J. Ren, and J. A. Edmond, J. Vac. Sci. Technol. B 14, 2349 (1996).

[3] E. S. Hellman, D. N. E. Buchanan, and C. H. Chen, MRS Internet J. Nitride Semicond. Res. 3, 43(1998).

[4] A. Bourret, A. Barski, J. L. Rouvière, G. Renaud, and A. Barbier, J. Appl. Phys. 83, 2003 (1998).

[5] S. W. King, C. Ronning, R. F. Davis, M. C. Benjamin and R. J. Nemanich, J. Appl. Phys. 84, 2086 (1998).

[6] H. P. D. Schenk, G. D. Kipshidze, U. Kaiser, A. Fissel, J. Kräusslich, J. Schulze, and W. Richter, J. Cryst.Growth 200, 45 (1999).

[7] V. Lebedev, B. Schröter, G. Kipshidze, and W. Richter, J. Cryst. Growth 207, 266 (1999).

[8] C. S. Davis, S. V. Novikov, T. S. Cheng, R. P. Campion, and C. T. Foxon, J. Cryst. Growth 226, 203 (2001).

[9] N. Onojima, J. Suda, and H. Matsunami, Appl. Phys. Lett. 80, 76 (2002); J. Cryst. Growth 237-239, 1012 (2002).

[10] A. R. Smith, R. M. Feenstra, D. W. Greve, J. Neugebauer, and J. E. Northrup, Phys. Rev. Lett. 79, 3934 (1997).

[11] C.D. Lee, Y. Dong, R.M. Feenstra, J.E. Northrup, J. Neugebauer, Phys. Rev. B 68, xxxx (2003).

[12] V. Lebedev, B. Schröter, G. Kipshidze, and W. Richter, J. Cryst. Growth 207, 266 (1999).

[13] G. Koblmueller, R. Averbeck, L. Geelhaar, H. Riechert, W. Hösler, and P. Pongratz, J. Appl. Phys. 93, 9591 (2003).

[14] J. E. Northrup, R. Di Felice, and J. Neugebauer, Phys. Rev. B 55, 13878 (1997). 